\title{
Summary of the Mini BNL/LARP/CARE-HHH Workshop on Crab Cavities for the LHC
}

\section{(LHC-CC08)}

I. Ben-Zvi, R. Calaga, F. Zimmermann

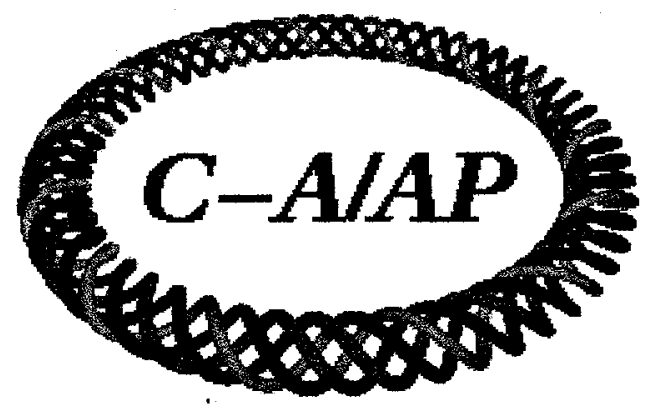

\section{Collider-Accelerator Department Brookhaven National Laboratory Upton, NY 11973}

Notice: This document has been authorized by employees of Brookhaven Science Associates, LLC under Contract No. DE-AC02-98CH10886 with the U.S. Department of Energy. The United States Government retains a nonexclusive, paid-up, irrevocable, world-wide license to publish or reproduce the published form of this document, or allow others to do so, for United States Government purposes. 


\section{Summary of the Mini BNL/LARP/CARE-HHH workshop on crab cavities for the LHC (LHC-CC08)}

Editors: I. Ben-Zvi, R. Calaga, F .Zimmermann April 28, 2008 


\title{
1 Summary of LHC-CC08
}

\author{
I. Ben-Zvi, R. Calaga, F. Zimmermann
}

\subsection{Introduction}

The first mini-workshop on crab compensation for the LHC luminosity upgrade (LHC-CC08) was held February 24-25, 2008 at the Brookhaven National Laboratory. A total of 35 participants from 3 continents and 15 institutions from around the world participated to discuss the exciting prospect of a crab scheme for the LHC. If realized it will be the first demonstration in hadron colliders. The workshop is organized by joint collaboration of BNL, US-LARP and CARE-HHH. The enormous interest in the subject of crab cavities for the international linear collider and future light sources has resulted in a large international collaboration to exchange aspects of synergy and expertise. A central repository for this exchange of information documenting the latest design effort for LHC crab cavities is consolidated in a wiki page: https://twiki.cern.ch/twiki/bin/view/Main/LHCCrabCavities

\subsection{Workshop Objectives}

The main goal of this workshop was to define a road-map for a prototype crab cavity to be installed in the LHC and to discuss the associated R\&D and beam dynamics challenges. The diverse subject of implementing the crab scheme resulted in a scientific program with a wide range of subtopics which were divided into 8 sessions. Each session was given a list of fundamental questions to be addressed and used as a guideline to steer the discussions. The 8 sessions and their associated charges were:

- Day I:

- Session I: Introduction \& Overview

- Session II: Optics \& LHC Integration

* Choice of Freq: $800 \mathrm{MHz}(400 \mathrm{MHz}$ )

* How much free space is available both for global or local scheme?

* Global or local schemes for Upgrade Phase I ?

- Session III: Crab Cavity Noise Effects

* Noise effects and simulation benchmarking status

* Operational reality

- Session IV: Design, Fabrication \& Processing Challenges

* Max achievable gradient in deflecting mode

* Required length of the crab system (cavity cryostat etc..)

* Do we need more than 2-cells (pros-cons)

* How much polarization is need (beam related, space related)

* Optimum geometry and cavity aperture

* Damping mechanisms (Effective + Robust)

- Day II:

- Session I: Other Crab Cavity R\&D in the World

* Lessons to be learned from previous and ongoing projects

* What components can be directly adapted for the LHC system

* Which components need most R\&D focus 
- Session II: Impedance Issues

* Cavity impedance and frequency choice

* Cavity Aperture

* HOM power and efficient extraction mechanisms

* Thresholds for single and multi bunch effects

- Session III: RF Control

* Input power requirements \& Qext

* Power handling capacity of the different couplers

* Max. allowable phase jitter, feedback requirements

* Type of power amplifier and related stability

- Session IV: Discussion \& Work Packages

* Main R\&D steps and priorities

* Distribution of work packages among the constituents

* Rough time scales

\subsection{Some Key Conclusions of the Workshop}

- Choice of Freq:

- $800 \mathrm{MHz}$ is best for Phase 0, lower frequencies are preferred ( $\leq 400 \mathrm{MHz}$ ). Need for more beam-beam simulations including RF curvature is crucial.

- Gradient of 2.5-3 MV for 2 cell $800 \mathrm{MHz}$ cavity $\left(\mathrm{E}_{\text {peak }}<40 \mathrm{MV} / \mathrm{m}, \mathrm{B}_{\text {peak }}<120 \mathrm{mT}\right.$ )

- 1-2 crab structures/beam should be sufficient. Additional degrees of freedom from optics

- 0.75 squash ratio is reasonable to fabricate and will fit for new optics with VV crossing

- Cavity aperture $>10 \mathrm{~cm}$ diameter

- Various designs of couplers available, beam pipe coax + waveguide may be most effective and robust

- Choice of cavity structure:

- Exotic cavity shapes were proposed and they may offer at least a simpler local crab scenario, possibly even a lower operating frequency

- Resolve peak surface fields and multipacting issues to use a compact cavity shape

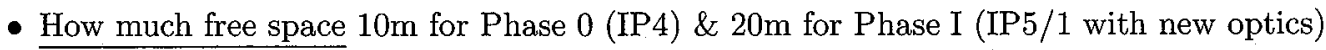
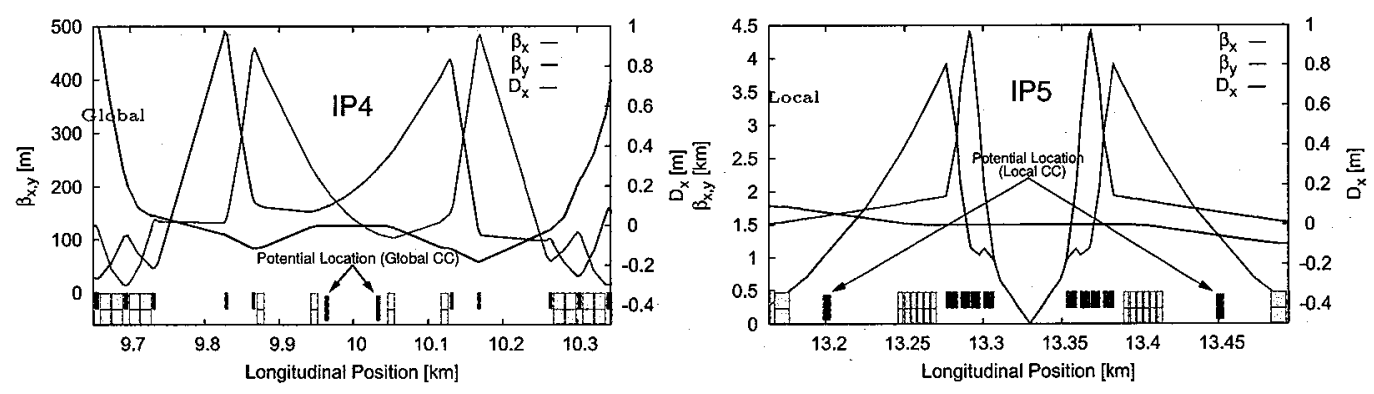
- Global or Local Phase I

- Collimation has to evaluate the exact loss maps and additional heat deposition from oscillating bunch in the global scheme. Configuration must allow for an additional $0.5 \sigma$ orbit

- Can we optimize the existing collimators to exploit the oscillating bunch (longitudinal collimation) to reduce the impedance?

- Noise Effects: Need more strong-strong simulations to understand remaining issues but current estimates and RF jitter suggest that LLRF can keep the jitter within required tolerances

- R\&D Objectives

- Adapt from previous R\&D (ILC, light sources, etc..): LLRF, couplers, cryostat(LHC), tuners

- Focus priorities: collimation, impedance, final cavity design and couplers, common cryostat, simulations, measurement on models, and testing with beam

- Investigate the potential and feasibility of compact cavity shapes and associated peak surface fields and multipacting issues.

- Investigate the possibility to optimize the existing collimators setup to exploit the oscillating bunch (longitudinal collimation) and aim at a reduction of impedance

- Cavity impedance needs careful evaluation to establish the resulting single bunch and coupled bunch effects and specify requirements. Start with assumptions used for existing narrow band impedances in the $\mathrm{LHC}\left(\mathrm{R}_{s h}<200 k \Omega, \operatorname{Im}\left\{\frac{Z}{n}\right\}<0.15 \Omega, \mathrm{R}_{s h}^{T} \ll 2 M \Omega\right)$.

- $\underline{\mathrm{RF} \text { Control }}$

$-Q_{\text {ext }} 10^{5}-10^{6}$

- Power handling - beam pipe coax + ferrites robust for high currents

- Phase jitter control easily possible $\leq 1 \times 10^{-2} \mathrm{deg}$, needs $\leq 1 \times 10^{-3} \mathrm{deg}$ which is at the limit of the existing technology $(800 \mathrm{MHz})$.

- Power Amplifiers: IOT (50-100 kW)

A summary and essential details of each session including the detailed conclusions of the workshop charge are covered in the following sections which were drafted by the respective session conveners. 


\section{Day I, Session I: Introduction and Overview}

\section{F. Zimmermann}

The starting session of the joint BNL/LARP/CARE-HHH mini-workshop on LHC crab cavities featured four presentations. After welcoming the participants in the Brookhaven Center, the workshop chair Ilan Ben-Zvi described the workshop program and goals. Robert Palmer reviewed the history and motivation of crab cavities, and he also recalled some related, possibly less known ideas. Rama Calaga presented a thorough overview of LHC crab-cavity schemes, highlighting challenges and outstanding questions. Finally, Mike Cole introduced the SBIR proposal for an $800-\mathrm{MHz}$ crab-cavity prototype.

Ilan pointed out that BNL was a good place for an LHC crab workshop, as BNL physicist Robert Palmer did not only invent the crab cavities, but also the two-in-one dipole, which is a key element of the LHC project. He then listed the workshop goals, which included (1) to learn from KEKB, (2) to draw the road-map for LHC crab cavities with time line and time scales, and (3) to define work packages and distribute tasks. A number of decisions also needed to be taken: choice between global or local scheme, optics, small-angle straw man, requirements for local scheme, exotic cavities, RF frequency, D1 magnet parameters, engineering challenges, $R \& D$ plan, and prototype cavity. The most important question to be addressed was "how do we get started?" and should we plan for an installation in LHC IR4?

Robert Palmer next guided the workshop through the history of the crab-cavity concept, which he had originally proposed for linear colliders in 1988. Another idea which he developed a few years earlier is "super-disruption" where pre-collisions with transversely large lower-energy electron (or positron) bunches act as powerful dual-plane focusing lenses which could yield unprecedentedly small effective beta functions at the primary IP. For electron-positron colliders this idea was not further pursued in view of the Oide limit. Since for protons synchrotron radiation is a much weaker effect, the super-disruption concept might, in principle, work for the beams circulating in the LHC. The focusing collisions with electron beams can be facilitated by applying crab cavities for all proton and electron beams involved, giving rise to "crab-super-disruption". Additional proton-electron collisions would be required on the outgoing side, after the primary proton-proton collision, in order to match the proton beams back into the ring. It is interesting that originally neither crab cavities nor super-disruption had been considered for a ring. More information can be found in three references quoted by Bob.

Rama Calaga presented a draft road-map for LHC crab-cavities, where different crab-cavity stages coincide either with the nominal LHC or with one of two proposed upgrade phases. Optimistically, a stage- 0 crab cavity could be installed in IR4 by 2010-11, and a local crab system be implemented together with the phase-1 IR upgrade presently foreseen for 2012. On a longer horizon an advanced phase-2 upgrade of the LHC IRs with associated major crab modifications might happen around 2016-17, at the earliest.

Crab cavities will yield about $15 \%$ luminosity gain for the nominal LHC, and upto a factor 2-3 higher luminosity for the LHC upgrades. For a "global" crab scheme, crab cavities are installed at one or two places in the ring, far from the high-luminosity collision points in which the crabbed bunches should collide. A preferred location for global crab crossing is in IR4 close to the existing accelerating RF cavities, since this region offers the largest separation between the two beam lines $(42 \mathrm{~cm}$ instead of the standard $19 \mathrm{~cm}$ separation). For the nominal LHC, the beta functions in this region have a magnitude between hundred and a few hundred meters. Katsunobu Oide remarked that the beta function at a global crab cavity in IR4 could be increased to $800 \mathrm{~m}$ with a minor optics change. In the global scheme, the closed orbit all around the ring depends on the longitudinal position with respect to the bunch center.

In the case of vertical crossing in one IP and horizontal crossing in another, two global crab cavities per beam and appropriate phase advances between IPs would be required for crabbing in both IP1 and IP5. The local crab scheme corresponds to the original idea. Here, bunches are crabbed prior to reaching IP1 or IP5 and "un-crabbed" on the other side of each collision point. Potential local crab-cavity locations are found between the D1 and D2 separation dipoles, where the beta functions, for a beta-star of 0.25 
$\mathrm{m}$, amount to a few $\mathrm{km}$. For both the local or global scheme the crab voltages required vary between 2 $\mathrm{MV}$ and $10 \mathrm{MV}$, assuming a crab RF frequency of $800 \mathrm{MHz}$. These voltage values are of the same order of magnitude as what has already been demonstrated by the KEKB crab cavities, which provide up to 2 MV at $500 \mathrm{MHz}$.

Rama stressed that due to transverse place constraints vertical-vertical crossing at the two main IPs may be the only possibility for the local crabbing scheme. An integrated cryostat for the two beams would almost certainly be required. Turn-by-turn random noise tolerances for the crab RF phase have been calculated in weak-strong and strong-strong beam-beam simulations as well as using analytical formulas for the transverse emittance growth. The obtained tolerances appear within reach of present $\mathrm{RF}$ technology. In particular, it is reassuring that the noise spectrum measured at the KEKB crab cavities would be acceptable for the LHC. Mike Cole presented the SBIR proposal that was recently submitted to $\mathrm{DOE}$, and which has as its primary objectives to design, fabricate and test an $800-\mathrm{MHz}$ prototype crab cavity for LHC, and also to generate a preliminary cryomodule design. The SBIR program is phased, extending over a total of 2.5 years, and it will be executed in a collaboration of Advanced Energy Systems, a company on Long Island, with BNL. The concept of the global crab scheme was not transparent to some of the participants. Amos Dexter explained that the global scheme works since "closed orbits" exist.

Frank Zimmermann reminded the audience that the global-crab scheme is in operation at KEKB, so that there should be no reason for doubt the underlying principle. The "z-dispersion" introduced by the crab cavity can be thought of in analogy to the regular momentum dispersion. Responding to a question by Ralph Assmann whether phase-0 could benefit both IPs, Katsunobu pointed out that with about $40 \%$ higher crab voltage and proper phase advance (e.g. 45/135deg to IP1 and IP5) a single crab cavity could be used to correctly crab the beam in both IPs, provided the plane of crossing is the same. Robert Palmer and Hasan Padamsee expressed concern about the short-range crab-cavity wake fields that could lead to "banana effects," as are seen in linacs.

A related phenomena would be the effect of collimator wake fields for the global crab scheme. Collimator wake fields would be excited by tilted bunches passing between collimator jaws. A self-consistent calculation will be needed to determine the magnitude and consequences of wake fields for the effective crab angle and bunch shape. Also, further strong-strong beam-beam simulations should explore the effect of the crab RF curvature. Several participants stressed that the crab-cavity parameters are similar for the local and global schemes. A discussion by Hassan and Frank Zimmermann indicated that the noise issues could possibly be relaxed for the local scheme. Crab super disruption can potentially be implemented as variant of a future "LHeC" collider.

John Byrd proposed to consider introducing dispersion at an RF cavity for an initial "poor man's" crabbing test, instead of installing a real crab cavity. His subsequent home work assigned by Katsunobu showed that one or two orders of magnitude (in either dispersion and/or RF voltage) are missing for such scheme to work at the LHC. A second proposal by John Byrd was the passive operation of the first crab cavity test exploiting beam loading and adjusting the transverse orbit through the crab cavity. A passive. test would save the initial costs for an RF generator and associated items. An independent cooling system for easier exchange of the crab cavities was discussed by Joachim Tuckmantel and Hassan. Fritz Caspers, participating remotely, suggested to perform beam tests of hadron-beam noise sensitivity in the CERN Antiproton Decelerator (AD). 


\title{
3 Day I, Session II: Optics \& LHC Integration
}

\author{
K. Oide
}

\subsection{R. Tomas}

- New idea from S. Fartoukh: move D2! Good for CCs.

- However phase I IR upgrade optics are not ready.

- Separation of beams to $27 \mathrm{~cm}$ for $20 \mathrm{~m}$ longitudinally achievable with present technology.

- CCs have an impact on particle stability

- Further studies with Beam-Beam required

- Betas at IP4 CC cannot be increased beyond $320 \mathrm{~m}$ due to aperture constraints with current configuration, but with inverted polarity on one of the quadrupoles, it can be increased to approximately $800 \mathrm{~m}$.

- Large phase tunability using arcs.

\subsection{R. Assmann}

- The LHC collimators must sit very tight on the beam to provide good passive protection and cleaning.

- As a consequence, the 6D phase space must be well defined. Tolerances on relative settings (retraction) are critical.

- Off-momentum beat is important and is being addressed (S. Fartoukh). Larger off-momentum beta beat with upgrade optics.

- A global crab cavity scheme will further complicate the situation, probably to the point where collimation breaks down.

- Tests with a global crab scheme can be performed with a few nominal bunches (increase of specific luminosity).

- Presently, little hope to improve integrated luminosity with global crab scheme.

- Further work is ongoing and required. Interference local crab cavities and collimation in experimental insertions.

\subsection{Y. Morita}

- High beam currents $(1.7 / 1.35 \mathrm{~A})$ stored with crab cavities.

- No serious beam instability caused by LOM/HOM. 
- HOM power successfully absorbed up to $12 \mathrm{~kW}$ in the ferrite dampers.

- Physics run with CRAB ON with high beam currents (1.62/0.95 A).

- LER crab voltage degraded to $1.1 \mathrm{MV}$, which was still applicable by increasing beta-x at the LER crab cavity.

- Crab phase was well controlled, although the LER tuner phase fluctuates.

- The beam oscillation observed with high current crabbing operation. It can be avoided by shifting crab phase by +10 deg.

- Trip rate during the physics run $0.4 / 3.5$ par day (last year). Trip rate of the HER cavity is less than 1 /day this year.

- KEKB crab cavities have been working with high beam currents to conduct physics run with the crab crossing.

\section{$3.4 \quad$ J. Tuckmantel}

- In the medium time range (having reasonable luminosity, beam current $\leq$ nominal) there is a good chance for a not permanently installed TEST CRAB CAVITY in the area around P4: at (missing) $\mathrm{ACN}$ or $\mathrm{AD}$.

- The additional hardware installation is manageable but not for free (cryo-lines, RF high-power equipment; controls).

- Apparent 'details' have to be settled with concerned LHC groups/persons even before starting construction of C.C. (i.e. before signing any contract).

Issues and Comments

- Besides the optics, the local scheme needs more attention on the required space for the rf system and cryogenics (K. Mess).

- It may be possible to increase the IR4 beta up to $800 \mathrm{~m}$ by switching the polarities of 2 quadrupoles, making symmetrical around the IP.

- The chromatic beta-beat should be cured by additional families of sextupoles, even for nominal operation before CC.

- Placing collimators only at non-beating section may solve the issue, though it is only possible in future.

- If the collimation has been already messed up without crab, does global crab really have an impact ?

- Further study on each issue experienced at KEKB may contribute to LHC-CC, including:

- degradation of the voltage

- cause of trip

- nature of the phase noise

- further analysis of the high-current oscillation 


\section{- tuner motion}

- A compensation of the banana effect by loading of the crab cavity may be studied for the $800 \mathrm{MHz}$ option, similarly to a compensation of the energy within a bunch in a linac. 


\title{
4 Day I, Session III: Crab Cavity Experience \& Noise Effects
}

\author{
A. Seryi
}

Two talks were given at this session, "The First Experience of Crab Crossing at KEKB" by Oide-san and "Synchro-Betatron Resonances \& Noise Effects" by Ohmi-san.

KEK-B has $22 \mathrm{mrad}$ crossing angle. Crab-cavities were considered as a way to increase luminosity of KEK-B. According to early simulations the beam-beam parameter was expected to increase to 0.15 with crab cavities (in comparison with 0.06 without). It was expected to gain more than just the geometrical overlap - with crab cavity the collision is effectively head-on, and with horizontal tune close to half integer the dynamics become effectively one dimensional (plus synchrotron motion) as the beam-beam force becomes nearly independent on the horizontal coordinate.

A single crab cavity scheme per ring was implemented at KEK-B (beam tilts all around the ring) to save the cost of cavities and cryogenics. Various observation and measurements were made to characterize the crab cavity. In particular, phase stability of the crab mode was measured and found to be better than the requirements. Slow stability below $1 \mathrm{~Hz}$ was about 0.02 and 0.01 degrees for LER and HER correspondingly.

Independent measurement by a spectrum analyzer have shown better than 0.01 deg for $f>2 \mathrm{kHz}$ and $0.1 \mathrm{deg}$ for $2 \mathrm{~Hz}<\mathrm{f}<2 \mathrm{kHz}$. Several distinct frequencies were observed in the spectrum, in particular 32 and $64 \mathrm{kHz}$ as well as $32,37,46,50$ and $100 \mathrm{~Hz}$. In further discussion after the talk it was noted that harmonics near hundred $\mathrm{Hz}$ may be due to mechanical vibration of cavity, $13 \mathrm{~Hz}$ due to vibration of coaxial coupler, and $32 \mathrm{kHz}$ perhaps due to switching power supply.

Tests of the crab cavity and of the restored head-on collision included various measurements with beam, such as measurements with streak camera, scan of phase and crab voltage, etc. - they all confirm the effective crabbing. The effects on the luminosity and beam-beam parameter have shown that the highest achieved vertical beam-beam tune-shift parameter was about 0.088 , while without crab it was 0.055. The specific luminosity increased considerably, and more than the geometrical gain, however the measured luminosity corresponded to simulations only at low currents. There are various ideas why the specific luminosity drops faster than expected, but all of them require further investigation.

Synchro-beta resonances were studied with respect to the noise in beam-beam interaction at LHC by Ohmi-san. For comparison, KEK-B case with $22 \mathrm{mrad}$ crossing angle was recalled, where the performance was degraded by about a factor of two at a high beam-beam parameter. Synchro-betatron resonances in LHC were studied in simulations and it was found that, with $0.15 \mathrm{mrad}$ half-crossing angle, degradation of luminosity and lifetime become noticeable only at $\times 8$ of the nominal bunch charge, while crab-cavities, which would restore head-on collisions, would eliminate the degradation. Large Piwinski angle cases $(2-3.5$, while nominal is 0.4$)$ were studied and no significant luminosity degradation was observed. (All these studies were performed without parasitic collisions).

Fluctuations of the collision offset caused by crab cavity noise, RF cavity noise or noise of the bunch by bunch feedback system can be detrimental as they would enhance diffusion. These effects were studied in high statistics weak-strong and strong-strong simulations, taking into account correlation time of the noise. Results of the strong-strong simulations are tighter than those of weak-strong, and show that the noise level of less than $0.1 \%$ is required for the turn by turn noise if the emittance doubling time is to be limited to $1 \times 10^{9}$ turns. However, it needs to be noted that simulations contain numerical offset noise of about $0.1 \%$ and higher statistics would be difficult to achieve. Simulations also show that the tolerance becomes about $\sim 1 \%$ for noise with correlation of 100 turn. 


\title{
5 Day I, Session VI: Design, Fabrication \& Processing Chal- lenges
}

\author{
H. Padamsee
}

\subsection{KEK Crab Accomplishment Summary}

KEK has successfully demonstrated the operation of two crab cavities ( $509 \mathrm{MHz}$ ) above $1.44 \mathrm{MV}$ kick, with high beam current to observe crabbing (beam deflection), head-on collision, increase of vertical tune shift, and increase of specific luminosity per bunch for low bunch current. At higher bunch currents, the specific luminosity falls faster than simulations for head-on, so that the hoped-for increase of total luminosity is not yet observed. This is possibly due to many effects which are under investigation.

Vertical, horizontal and beam tests all exceeded design values for crab kick $(1.4 \mathrm{MV}$, Epk $=21 \mathrm{MV} / \mathrm{m}$ and $\mathrm{Hpk}=64 \mathrm{mT}$ ). The trip rate fell from 3 per day to 1 per day over 4 months of operation and learning. Some troubles with coaxial coupler cooling and the tuner still need work. The kick has fallen to $1.1 \mathrm{MV}$ due to these troubles.

In getting to the successful demonstration several challenges were encountered and addressed with the coaxial coupler and tuners.

The overall length of the KEK module is about $3 \mathrm{~m}$.

\subsection{Summary of Discussion for LHC Crab Options}

Several options were discussed for the first demonstration of crab cavities in LHC.

- One crab cavity in one ring for global crabbing. This project would emphasize the development and testing of the cavity and cryomodule without significant immediate benefits to luminosity and with minimal information about beam-beam interactions. Emittance growth and HOM extraction would be studied.

- Two crab cavities in the global crabbing mode (1 per beam) to reach a crossing angle of 0.5 mrad. Here it would be possible to get information on the beam-beam interactions in head-on collisions and possibly $10-15 \%$ gain in luminosity if all goes well. The increased luminosity would make it more attractive for LHC to support the installation. The small increase in luminosity however may be difficult to confirm.

- Four crab cavities in the global mode ( 2 per beam, 1 per plane) to benefit two interaction regions. Given the developmental aspects that need to be addressed this option is more expensive and would need more time to implement. The potential benefit to two interaction regions would probably generate more support for installation.

- Two crab cavities per beam in the local position. This option would have to address the tighter space availability near the IPs. The geometry of polarization can be used to accommodate the 800 $\mathrm{MHz}$ cavity. A common crab cavity design that works for both local and global positions would be best to minimize repetition of the development/prototype effort.

Practical considerations such as the availability of refrigeration may influence the choice of local or global positions.

The aperture (depending on upgrade IP design) is constrained to approximately $8 \mathrm{~cm}$ diameter. It would be wiser to design a cavity with a larger aperture. The need to extract HOMs will also lead to larger apertures. 
The highest frequency compatible with aperture and $\mathrm{rf}$ curvature considerations is $800 \mathrm{MHz}$. A lower frequency would face space challenges, especially for the local crabbing option, and need more crab voltage for a given crossing angle.

\subsection{Crab kick needed}

At $800 \mathrm{MHz}$ a crab voltage of $2 \mathrm{MV}$ would deliver the needed crossing angle (1 mrad) for the proper location and lattice (choice of beta at the crab position).

\subsection{Design Options}

\subsubsection{Single cell}

As a default option, using the same surface fields as the KEK crab cavity (f $=509 \mathrm{MHz}, \mathrm{Epk}=21$ $\mathrm{MV} / \mathrm{m}, \mathrm{Hpk}=60 \mathrm{mT}$, kick $=1.44 \mathrm{MV}$ ) and scaling the cavity to $800 \mathrm{MHz}$ gives a kick voltage of 0.9 MV per cell. If we use technology improvements to raise surface fields by a factor of 2 (to Epk $=40$ $\mathrm{MV} / \mathrm{m}, \mathrm{Hpk}=120 \mathrm{mT}$ ), the KEK single cell design single cell will provide $1.8 \mathrm{MV}$ crab kick. This is close to the needed kick. The cell design can be further optimized to lower surface fields. The operating temperature has to be $2 \mathrm{~K}$ due to the higher frequency. Paths to refrigeration need to be developed.

\subsubsection{Two-cell}

The 2-cell cavity offers two benefits. Same surface fields as for KEK, or higher crab kick. Strong HOM damping should still be possible with 2-cells through 2 beam tubes. Lower surface fields are better for reduced trip rate. LHC is very sensitive to trip rate because fill time is very long.

\subsubsection{LOM coupler}

This is the source of many troubles both during development and operation. A waveguide coupler is an alternative for the coaxial LOM coupler. A choke filter is still needed for the crab mode. Significant development work is needed for the change.

\subsubsection{Exotic Structures}

A number of exotic shape ideas are brewing. Rod type structure has very small aperture. Magnetic field of TM010 mode needs careful study for beam loading and instability. Most participants considered exotic structure as option to pursue in parallel with baseline $\mathrm{TM}_{110}$ crab cavity. 


\section{Day II, Session I: Other Crab Cavity R\&D in the world}

\section{Ben-Zvi}

This session contained four talks covering crab cavity $\mathrm{R} \& \mathrm{D}$ from various (non $\mathrm{LHC}$ ) projects. The session did not cover the very extensive work done at KEK on the KEK-B crab cavities. That work was presented in a number of other sessions, including Monday session II , session III (completely dedicated to results from KEK) and Session IV. In the Tuesday session I, Andrei Seryi (SLAC) and Peter McIntosh presented work on the ILC crab cavities. Andrei provided an overview and requirements, Peter covered design and test results. Then Alireza Nassiri presented deflecting cavities for light sources, and Fabio Marcellini (INFN-LNF) talked about the RF deflectors at CTF3.

The objectives set for the session were:

- Lessons to be learned from previous and ongoing projects.

- What components can be directly adapted for the LHC system.

- Which components need most R\&D focus.

In the talk by Andrei Seryi, we learned about the comprehensive design made for the crab crossing of the ILC and the various constraints that shaped the design. The objective in the ILC is similar to that of the LHC, which is to introduce a crossing angle but minimize the associated loss of luminosity. An interesting lesson learned (which similarly affects the LHC design) is that even in the linear, large ILC, space at critical points is at premium, both along the beam path and orthogonal to it.

The ILC presents unique problems due to the fact that its beam is very flat. For example, this requires a very precise alignment of the crab cavity, to prevent feeding of crab angle from the large to the small dimension of the beam. We also learned that, given their experience, the SLAC ILC team is interested in getting involved in the design of a complete crab IP of the LHC.

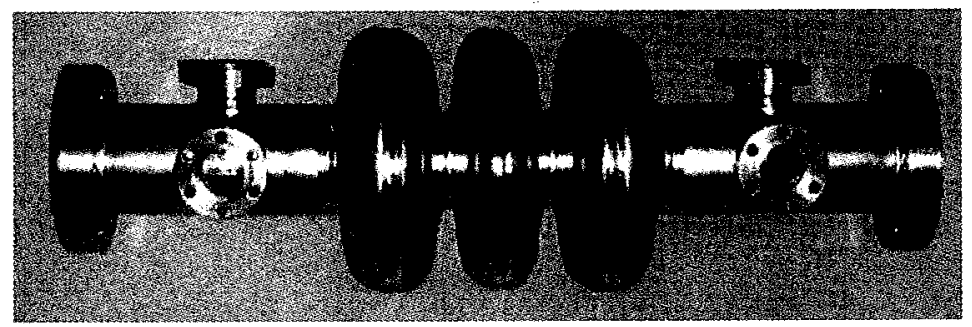

Figure 1: $3.9 \mathrm{GHz}$ crab cavity which achieved a crab gradient of $7.5 \mathrm{MV} / \mathrm{m}$ (FNAL)

Peter McIntosh listed (among other things) key technological challenges which must be met in the design, fabrication and installation of a crab cavity in the ILC:

- Damping and Couplers:

- Input coupler (based on DESY/FNAL 3rd harmonic),

- Lower Order Mode, LOM (issues of multipacting, tunability and fabrication),

- Same Order Mode, SOM (requiring very high damping, tunability),

- High Order Mode, HOM (with multipacting, tunability and fabrication challenges).

- Cryomodule: 
- Field polarization (1 mrad, issue which is more severe for the ILC's flat beam),

- Microphonics rejection (impacting RF power overhead, LLRF control),

- Cavity alignment (6 $\mathrm{nm}$ vertical beam size at IP, mostly ILC issue),

- ILC installation constraints (extraction beamline $18 \mathrm{~cm}$ away).

- Beam test verification:

- Verify cavity/wakefield design (single cavity),

- Verify LLRF and synchronization stability (single/dual cavity),

- Verify crabbing field polarization (single/dual cavity).

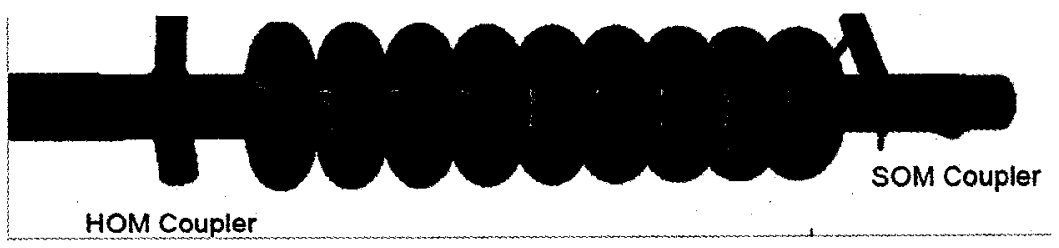

Figure 2: ILC 9-cell crab cavity showing the field intensity of the crab mode and the location of various couplers.

Ali Nassiri talked about a different application altogether from colliding bunches with minimal luminosity loss. Namely, at ANL the objective is to produce extremely short X-ray pulses from light sources with minimal loss in brightness. He presented a comprehensive overview of Deflecting Cavities for Light Sources, including the advantages to the users seeking short and intense X-ray pulses from existing light sources of the third generation, with a particular emphasis on the implementation of crab cavity short pulse generation at the Advanced Photon Source (APS).

The technique uses A. Zholents' scheme for a z-dependant deflection of the electron bunch, which is then introduced to an insertion device (wiggler or undulator) to produce an intense X-ray pulse which carries in it the transverse - longitudinal correlation of the electron bunch, followed by a tilted X-ray grating. The X-ray reflected by the grating in a particular direction uses the transverse-longitudinal correlation to get a very short X-ray pulse. In certain similarity to the ILC beam, the APS beam is also very flat.

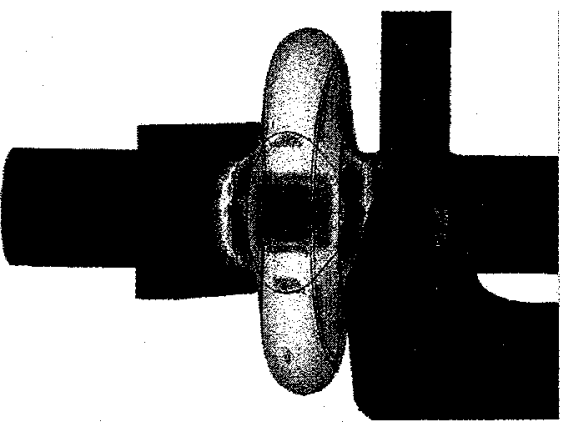

Figure 3: $2.8 \mathrm{GHz}$ Superconducting Single-Cell Deflecting Cavity considered for the APS short X-ray pulse generation. 
In the light-source short-pulse generation application, emittance increase is a driving consideration since the beam stays in the ring and must preserve its high brightness for all users. Again, we find that at the APS longitudinal space is also at premium.

A relevant result which is worth mentioning here (although presented in session M IV) is the construction and successful test at Jefferson Laboratory of a scaled deflecting cavity for the ANL. The construction of the cavity was reported by Larry Turlington and the results of the test were reported by Peter Kneisel.

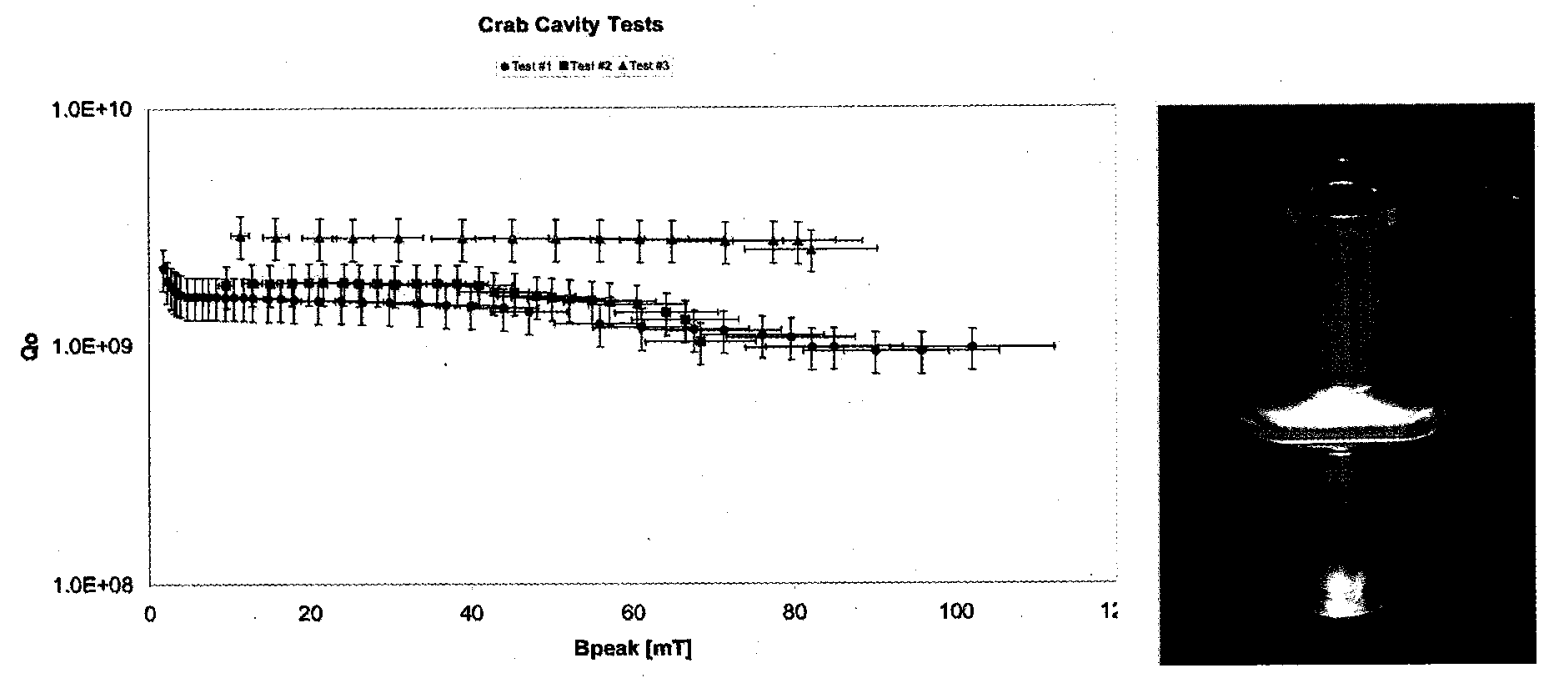

Figure 4: $2.8 \mathrm{GHz}$ prototype crab cavity for the APS and its Q vs. peak magnetic field plot from JLab tests.

Finally, Fabio Marcellini presented development and tests of RF deflectors at the CLIC Test Facility CTF3. This is yet a third unique application of deflecting-mode cavities.

The RF deflecting cavities of the CTF3 are used in two rings for injection/ejection of long trains of bunches. This operation increases the current of the bunch train by nearly a factor of 8 , while reducing the length of the train. The beams are produced in a linac, and the purpose of the rings is to reduce the bunch-to-bunch separation by a clever timing of the deflection, to achieve a factor two increase in the peak current in a "delay loop", followed by a factor of four increase in a "combiner ring".

The CTF3 uses normal-conducting pulsed cavities. The system works successfully, but a vertical instability was discovered which is now being studied.

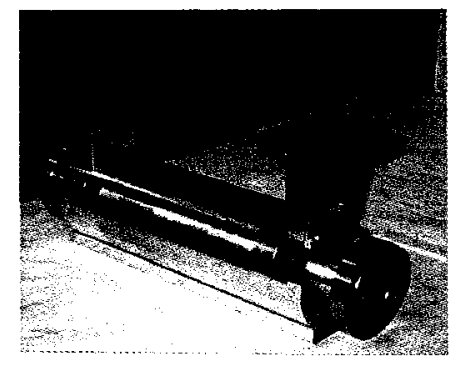

Figure 5: A CTF3 kicker cavity. 
This session did not have a discussion period. In conclusion:

- The most valuable asset the LHC CC has is the KEK experience. One should strive to add in areas that are unique to the LHC.

- The most valuable items to borrow from the experience of the ILC is the experience of teams in simulations, modeling, couplers etc.

- The various cavity shapes, couplers, tuners and cryostat design developed by the various efforts around the world can be used for cutting on the design time.

- Powerful simulations codes and expertise exist in various laboratories and should be used. 


\section{Day II, Session II: Impedance Issues}

\section{R. Rimmer}

This session contained three talks focused on impedance calculations and optimization of structures for HOM damping. Zhenghai Li (SLAC) talked about wakefields and design considerations, Graeme Burt (Cockcroft), presented impedance measurements with the ILC prototype and Derun Li (LBNL) talked about couplers for LOM and HOM damping. Zenghai began by introducing the suite of analysis codes developed at SLAC under the SciDAC program. These codes have been well benchmarked and are capable of modeling large-scale problems with high precision. He showed examples including the optimization of the "squash" ratio of an elliptical crab cavity, and how the deformation of the shape tunes the HOM frequencies. Most notably for the squashed shape the same-order mode (SOM) is tuned up in frequency so it may be damped without affecting the operating mode. The optimum squash ratio of about 0.8 is very similar to that chosen by KEK for the KEK-B crab cavity. Zhenghai also showed calculation of the surface fields for a 2-cell cavity and details of various damping schemes, both coaxial and using waveguides. One promising scheme utilizes a short coaxial damper followed by a coax-to waveguide transition. This should give strong damping in a compact structure. If applied to a squashed shape and if the dimensions of the coax are chosen appropriately all unwanted modes may be damped by this configuration.

Graeme showed detailed calculations for the ILC 9-cell crab cavity and extensive bench measurements on an aluminum model. Both bead-pull and wire measurements were used to verify mode identity and strength. Several types of HOM and LOM coupler were tested. The cells are basically round in this design with only small perturbations introduced to break the dipole mode symmetry. The results were in broad agreement with simulations and look promising for the ILC specifications. Graeme also showed some concepts for damping a 2-cell cavity as an option for LHC. The damping requirements for LHC are expected to be much more stringent than for ILC.

Derun showed a range of simulations for various damping methods on single-cell and multi-cell cavities studied for LUX, ALS, APS and a separator cavity for ANL. These results included collaborations with ANL, JLab and Tsinghua University in Beijing. He also presented results of measurements made at Tsinghua on a 3-cell aluminum model. Once again there was good agreement between simulations and measurements.

Discussion included debate over the space constraints and overall requirements for either a local or global scheme. The contribution to the broad-band impedance is not expected to be significant. The impedance is dominated by collimators etc.., but the narrow band impedances due to cavity modes could be a significant concern. An impedance budget was not presented to the meeting and this is critical to assessing all the various schemes that have been proposed. A reference was made to a paper by Daniel Boussard at PAC 99 [1], that contains a few relevant numbers, and the subject is discussed in the LHC conceptual design report [2], but this references other papers for the details of the mode damping requirements. Other references include $[3,4]$. Once this information is distilled it will be possible to evaluate the competing designs and see if multi-cell cavities are practical or whether individually damped single cell cavities are required. A word of caution here, there are multiple conflicting definitions of longitudinal and transverse impedance and any future analysis should state clearly which definition is being used.

It was clear from all of the presentations that there are a variety of simulation tools available to model the strongly-damped crab cavity and they have been reasonably well benchmarked against each other and against measurements. However to get accurate results requires some level of expertise and experience. In addition practical hardware such as HOM loads etc. are not perfect and the damping achieved in reality may not be the same as the numerical model or bench measurement. For these reasons a generous safety factor should be included in any analysis of the stability threshold for LHC. At least an order of magnitude would be advisable. Introducing a beam instability in LHC during the initial test would be 
very detrimental to the program.

The good news is that there are a lot of alternative designs that may meet the requirements and a number of groups are still exploring these and other options. Once the impedance budget and other constraints are made available these concepts can be evaluated against common criteria and the most promising can move forward to look at an integrated solution. The goal should be to achieve this before the next LHC crab cavity meeting (tentatively proposed for October 2008).

\section{References}

[1] D. Boussard, E. Chiaveri, E. Haebel, H.P. Kindermann, R. Losito, S. Marque, V. Rdel and M. Stirbet, "The LHC Superconducting Cavities", Proceedings of the 1999 Particle Accelerator Conference, New York, 1999. http://cern.ch/AccelConf/p99/PAPERS/MOP120.PDF

[2] "The Large Hadron Collider - Conceptual Design", CERN/AC/95-05 LHC.

[3] D. Brandt, L. Vos, D. Angal-Kalinin, "Intermediate Review of Single Bunch Collective Effects in the LHC", Proceedings of EPAC 2002, Paris, France. http://cern.ch/AccelConf/e02/PAPERS/MOPLE048.pdf

[4] D. Angal-Kalinin, L.Vos, "Coupled Bunch Instabilities in the LHC", Proceedings of EPAC 2002, Paris, France http://cern.ch/ab-abp-rlc-impedance/Literature/MOPLE047.pdf 


\section{Day II, Session III: RF Control}

\section{P. McIntosh}

This session contained 3 talks covering LLRF for Crab Cavities on ILC (by Amos Dexter, Cockcroft Institute), Amplitude and Phase Control (by Stefan Simrock, DESY) and finally Synchronization Activities at LBNL (by John Byrd, LBNL).

Amos started the session by highlighting the ILC crab cavity phase and amplitude specifications of $0.13^{\circ}$ and $2.2 \%$ in amplitude, with a synchronization tolerance of $<51 \mathrm{fs}$. Architectural options were identified utilizing a single phase locked LO (preferred) or otherwise utilizing ultra-stable dual LO's. An interferometer-based synchronization scheme would be chosen. He identified that the main ILC linac timing could not be used alone to provide the necessary synchronization at the IP. A phase control model, utilizing a PI-controller, has been developed which includes beam-loading, group delays, microphonics, phase detector responses and coupler characteristics. Optimization of this control model predicts $0.03^{\circ}$ and $0.3 \%$ stability. Problem will beam ON transients, which induce large RF power requirements, prefer Solid State Amplifiers (SSA) owing to their faster response times. If include $\pm 1 \%$ measurement errors, can predict $\pm 0.05^{\circ}$ and $0.7 \%$ stability. A prototype LLRF system has been fabricated utilizing low phase noise components (particularly phase detectors and mixers) and tests planned in April to verify performance utilizing $2 \times$ single cell SRF crab cavities.

Discussions that arose concerning application towards LHC; For the global scheme, the interferometer would not be required as only $1 \mathrm{CC}$ would be needed. Stefan Simrock commented that experience at FLASH had shown that the signals measured with beam are always much worse than predicted and that degradation in the phase reference system will degrade overall phase stability achievable, consequently dynamic calibration is an important requirement for these LLRF systems.

Stefan described the LLRF system development work performed at DESY for FLASH and XFEL. On FLASH, they are able to measure the $I$ and $Q$ response terms from the LLRF system. They observe cross-talk between the amplifiers and some $1 / \mathrm{f}$ phase noise contribution from a variety of sub-components. These features limit the field detection capability and deteriorate system performance. The sources of noise contribution have been characterized and grouped into areas; Reference containing $\mathrm{LO}$ and its timing distribution; Actuator and Drive containing VM and drive amp components, HVPS and klystron and DACs; Plant which contains cavity microphonics, beam loading etc and Field Detection which contains the phase and amplitude detectors and ADCs. Transient beam loading causes large gradient transients, can use feed forward techniques to improve stability. Another significant source of error is the vector sum calibration process. The pro's and con's for Analog vs Digital and the different types of control architecture were reviewed. Analog may in fact be preferred for LHC owing to the fact that for the global scheme it is only 1 cavity driven by a single amplifier, digital however would provide additional flexibility. Whatever the decision, the choice of electronic components is critical and need to optimize using low noise devices. $0.03^{\circ}$ and $0.01 \%$ achieved on FLASH, but with beam achieve $0.06^{\circ}$ but limited by diagnostics, may in fact be better than this! A klystron lineariser employed on FLASH to improve system response and the SRF cavities help in filtering the high frequency down-conversion noise inherent in the system.

Discussions that arose concerning application towards LHC: for the global system vector sum capability would not be required, making the architecture simpler. A single bunch EO detector could improve timing resolution ( $\sim 30 \mathrm{fs}$ achieved on FLASH). The main noise contributions are from $1 / \mathrm{f}, \mathrm{ADC}$, synchronization and LO and all need optimization to maintain system performance for LHC. Recommend 2 cavity probes to provide additional diagnostics. A question was raised concerning the beam ON transients expected on LHC and it was suggested that they should be similar to that at KEK-B. Oide reported that their LLRF system recovers quickly and that $1 / \mathrm{f}$ noise contribution is minimal.

John then described some of the ultra-fast synchronization studies underway at LBNL, comprising interferometrically stable fiber links. He showed that the system developed at LBNL has achieved <40fs 
stability over a $2 \mathrm{~km}$ 'dirty' fiber optic network on the LBNL site. LBNL are currently building a 16channel version for LCLS and initially achieved $<100 \mathrm{fs}$ stability over 1 wk and with thermal management improvements achieved 15fs over 1 month ( $<1 \mathrm{fs} /$ day drift). All measurements taken out-of-loop. John also reported that they are working on a fully integrated system of this type for FERMI@ELETTRA which includes the LLRF electronics. An RF LO signal has been superimposed onto the optical carrier which is then demodulated at the LLRF receiver to recover the RF synchronization signal. The same system architecture has been demonstrated at $476 \mathrm{MHz}$ and $2856 \mathrm{MHz}$ at SLAC. Discussions that arose concerning application towards LHC; The synchronization system would only be applicable for the local mode of operation on LHC. The LLRF system developed at LBNL is adaptable to LHC frequencies either at 400 or $800 \mathrm{MHz}$. 


\section{Day II, Session VI: Discussion \& Work Packages}

\section{R. Calaga, F. Zimmermann}

Due to the nature of the workshop a dedicated session for discussion to various key topics was scheduled. The main focus of this session was to discuss a draft road-map and identify the expertise and interest of collaborating institutes. This helped in division of the crab cavity tasks into respective subtasks assigned to the different collaborators. Due to time limitations some contributions to the workshop could not be accommodated in the regular sessions and thus were included as short contributions in this session.

Hasan Padamsee gave a short presentation on the large SRF facilities originally built to process and test $200 \mathrm{MHz}$ accelerating cavities to be used for neutrino factories and muon colliders.

A short presentation about phase noise thoughts by Fritz Caspers was presented by Frank Zimmermann. Phase noise is mainly dominated by the generator but the phase noise of the beam itself is many orders of magnitude larger. Another source of noise is conversion of AM-PM and viceversa. It was also noted that amplitude noise cannot be neglected and the subject seems to stress the effect of phase noise which may not be as delicate. An idea of using beating between two sine-waves instead of a single sinewave to reduce the blow-up results in time dependent crabbing which is not desired. It was also noted that the crab cavity affects the transverse phase space but sits on the revolution harmonic, hence cannot be responsible for transverse blow up since it sits far away from transverse Schottky bands. Finally, the upgrade scenarios of phase II (Early Separation, Large Piwinski Angle and Full Crab Crossing) were briefly reported from the IR07 workshop.

Ali Nassiri presented the various ANL capabilities like beam physics, simulation of RF structures, thermal analysis and available facilities for SRF R\&D. Their expertise and resources can aid in rapid development of a prototype for the LHC crab cavities and ANL is very interested in collaborating on various levels.

Leo Bellatoni via phone conference presented some thoughts on lower frequency cavities (200 $\mathrm{MHz}$ ) where the two counter propagating beams pass through the same cavity using the transverse deflecting electric field. It was noted that this kind of structure needs very strong damping which is under investigation.

Due to time constraints not all the session summaries were presented by the conveners. The final part of the workshop closed with discussion stimulated by the R\&D flow chart which includes the several sub-tasks and institutions involved to realize the prototype within a period of 3-4 years.

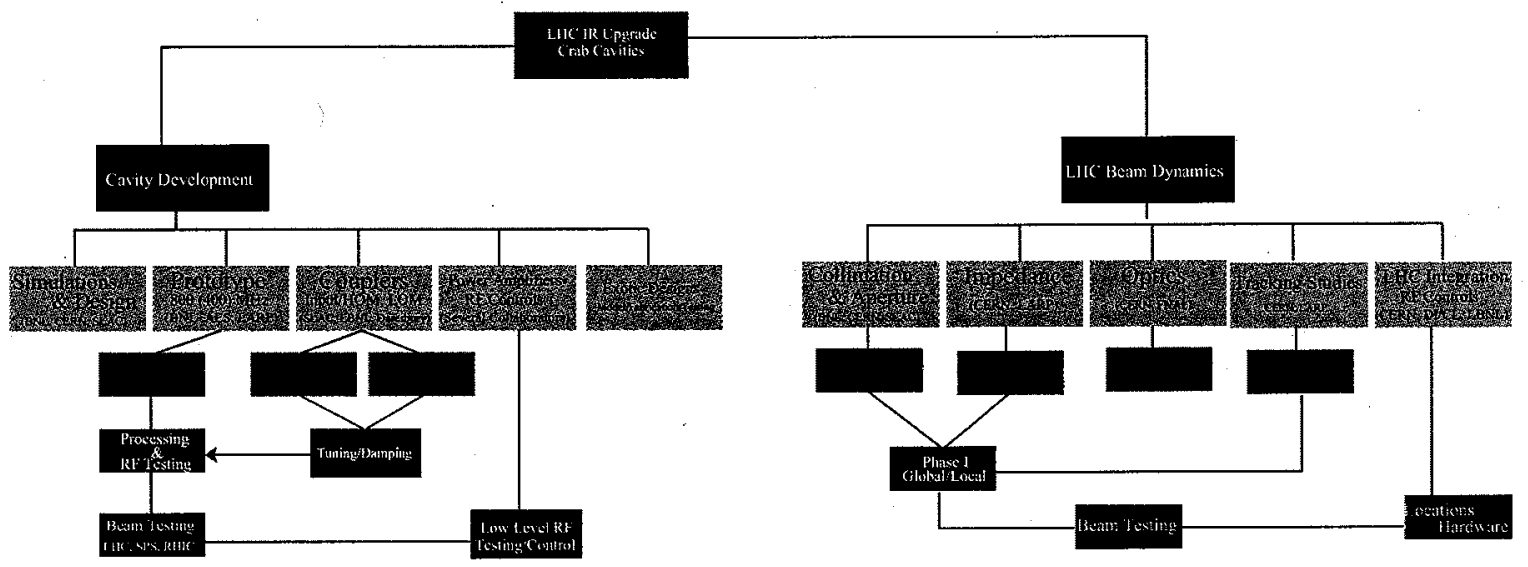

Figure 6: A schematic of R\&D for the LHC crab cavity prototype. 
The main conclusions from this session were:

- $\mathrm{R} \& \mathrm{D}$ of prototype to demonstrate $800 \mathrm{MHz}$ cavity goals and beam testing are crucial steps for a full scale crab cavity upgrade at the LHC.

- A modular approach exploiting the expertise of different interested collaborators is the most optimized way to build the prototype. Many components can be easily adapted from existing deflecting structures which will help to focus on LHC specific R\&D and achieve rapid progress.

- TWiki repository can aid as a central repository for efficient exchange of information.

- IP4 (RF sector) in the LHC shows the best promise for access to infrastructure and probability installation of the $800 \mathrm{MHz}$ prototype cavity.

- Various collaborators have committed (some loosely) to pursue the different R\&D items and to contribute man-power and resources towards the development and construction of the LHC crab structures.

The workshop's aim to establish a global collaboration was clearly achieved and the interest in the subject was unprecedented. The rapid progress and rising participation can be seen in figure below. It is anticipated that following the approval of the SBIR program to build the prototype, a design review of the cavity, couplers, cryostat and associated components will take place towards the end of 2008. Regular webex meetings to discuss the technical progress and to coordinate the efforts will be held and relevant information will be made available on the TWiki page.

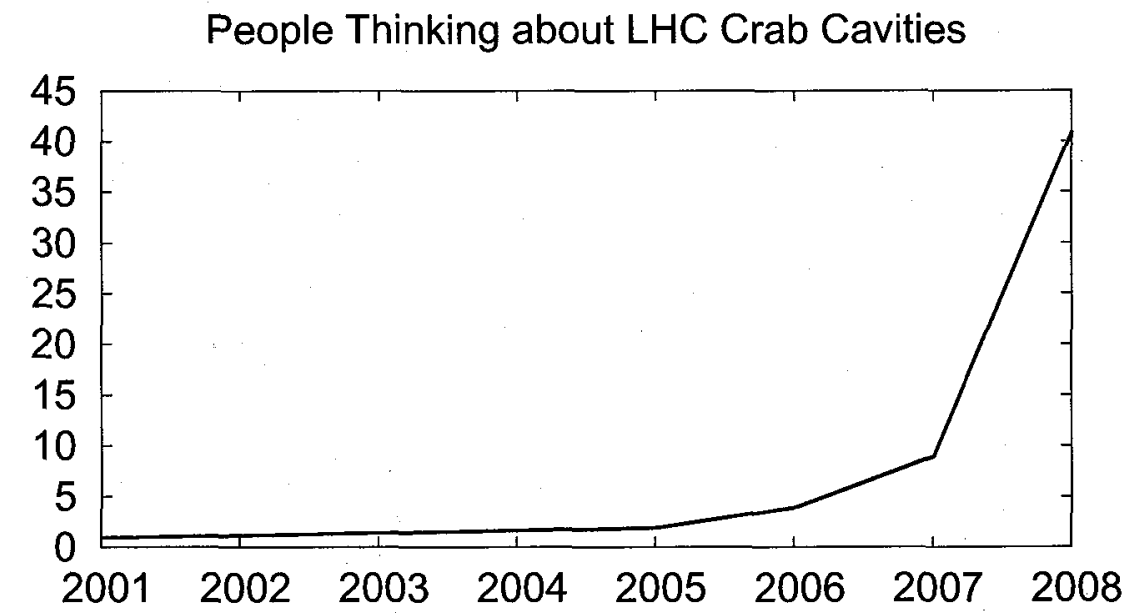

Figure 7: Number of people thinking about LHC crab cavities as a function of the year. 